\title{
Building a Knowledge Base System for an integration of Logic Programming and Classical Logic
}

\author{
Marc Denecker and Joost Vennekens \\ Department Computer Science, Katholieke Universiteit Leuven \\ Celestijnenlaan 200A, B-3001 Heverlee, Belgium \\ email: \{marc.denecker,joost.vennekens\}@ cs.kuleuven.be
}

\begin{abstract}
This paper presents a Knowledge Base project for FO(ID), an extension of classical logic with inductive definitions. This logic is a natural integration of classical logic and logic programming based on the view of a logic program as a definition. We discuss the relationship between inductive definitions and common sense reasoning and the strong similarities and striking differences with ASP and Abductive LP. We report on inference systems that combine state-of-the-art techniques of SAT and ASP. Experiments show that FO(ID) model expansion systems are competitive with the best ASP-solvers.
\end{abstract}

\section{Introduction}

Logic Programming (LP) with stable model semantics is commonly regarded as an effective KR system when used according to the ASP computational paradigm. Our position is that all the attractive features of the semantics and the ASP approach notwithstanding, there are alternative approaches that are better suited to address KR challenges, without compromising on computational adequacy. Our approach aims at a fundamental goal in the field of Knowledge Representation and Reasoning (KRR): to develop a Knowledge Base System (KBS), a system storing (declarative) domain knowledge and able to solve a range of tasks and problems in that domain, by applying various forms of inference on its knowledge base. As an example, imagine a KBS storing a specification of course scheduling at a university, and able to solve or support tasks of generating schedules at the start of the year, but also of verifying correctness of hand-made or revised schedules, of updating or revising the current schedule under additional or changed requirements, etc., all using the same KB.

The difference between the KBS paradigm and declarative programming frameworks such LP, ASP or Constraint Logic Programming (CLP), lies in the reuse of the $\mathrm{KB}$ for solving different problems and tasks requiring different forms of inference: deduction, model checking, model generation, update and revision, abduction, learning, etc. Thus, a KB does not encode a solution for a specific problem, nor is it a declarative program with an operational semantics induced by one specific form of inference. The $\mathrm{KB}$ is "only" a formal representation of declarative properties of the domain. This imposes a strong requirement on the KB language: its expressions should be interpretable as (informal) propositions about the domain, and this interpretation, its informal semantics, should be as clear, precise and objective as possible. First order logic (FO) is a language that satisfies this requirement by excellence. For example, if $H / 1, M / 1$ and 
$F / 1$ represent humans, men and women, then the sentence $\forall x(H(x) \supset M(x) \vee F(x))$ expresses the property that humans are men or (inclusive or) women. Our project uses an extension of FO with a concept whose informal meaning is understood with great precision throughout mathematics: the notion of an inductive definition. As we will argue, the resulting logic FO(ID) can be viewed as an integration of FO and LP.

Building a KBS is a compound research goal consisting of many subgoals which are challenging in their own right: development of KB languages and methodologies, building different sorts of inference systems, etc. Even when working on one of these subgoals, there are two reasons to keep the KBS paradigm in the back of our mind. The first is that the KBS paradigm focusses our research by putting constraints on the languages and systems to be developed. For example, the KBS paradigm induces us to work with truly declarative KR languages; it also induces us to study what knowledge is required to solve practical tasks and what forms of inference are needed for this. The second reason is that recent trends in computational logic suggest that building a useful KBS may not be as impossible as many believe now.

A fundamental constraint in building a KBS is the trade-off between expressivity of its KB language and efficiency of its inference engines. On the one hand, we need rich, expressive languages to specify in sufficient detail the background knowledge needed to solve real-world problems. On the other hand, expressive KB languages lead to computationally complex inference problems, which may be intractable or even undecidable. E.g., given FO's undecidability, there is no hope to use theorem proving for reliable problem solving in a full FO knowledge base. This consideration has led, e.g., the description logic area (DL) to build deductive knowledge bases for severely restricted versions of FO. The price is that in such languages, it is often hard or even impossible to specify the background knowledge required to solve many practical tasks.

The way out of this apparent deadlock, and the main hope for building practical applications using KBSs with more expressive KB languages, lies in the use of "cheaper" forms of inference, such as finite model checking, finite or bounded model generation or expansion, or approximate reasoning. In this respect, it is exciting to see recent trends in areas such as ASP, SAT and DL, that show that many real-world problems can indeed be solved by such forms of inference. ASP plays a prominent role in this evolution. It is based on the observation that deduction is often the wrong form of inference; what we often want as a solution for a task is (part of) a finite structure/interpretation satisfying a domain theory. In LP, the ASP paradigm was preceded by a somewhat similarly flavored paradigm based on abduction [1]. As stated in [2], the idea of finite model generation as a computational paradigm can also be applied for other languages than ASP. Indeed, some of the successful applications of SAT-systems, e.g., in planning, are by generating finite models as solutions to problems [3]. Other successful applications of SAT (e.g., in verification) are for problems of computing entailment or satisfiability of a domain theory in the context of a finite or bounded universe [4]. Another promising computational paradigm is approximate reasoning, which was developed and used recently for expressive description logics [5]. By integrating these forms of inference, a KBS system might be built that is able to solve a useful class of practical problems.

The next sections recall the main ideas underlying FO(ID), its role for KR and its relation to LP and ASP, and discuss the inference tools under development for this logic. 


\section{The KB-language FO(ID)}

In ASP, an answer set program is a sort of default theory [6]. From in the early days of LP, an alternative -but implicit- view was of a logic program as a definition; e.g., in completion semantics [7], or in datalog where intentional predicates are defined in terms of extensional ones. Many prototypical logic programs (e.g., member, append, transitive closure, etc.) are undeniably inductive (i.e., recursive) definitions. Also, the syntactical correspondence between LP and the way inductive definitions are often phrased in mathematics is more than striking. E.g.:

Definition 1. The satisfaction relation $\models$ of propositional logic is defined by induction on the structure of formulas:

$-I \models P$ if $P$ is an atom and $P \in I$.

- $I \models \varphi \wedge \psi$ if $I \models \varphi$ and $I \models \phi$.

- $I \models \neg \varphi$ if $I \forall \models \varphi$ (i.e., it is not the case that $I \models \varphi$ ).

This non-monotone inductive definition consists mainly of a set of informal rules, the third one with negation in the body. But the correspondence with logic programs goes beyond the syntactical level. Such an inductive definition defines a relation by describing how to construct it. The defined relation consists of all and only the tuples produced by a rule during this construction process. Thus, such inductive definitions consist of a set of informal rules augmented with a precise, natural, informal form of Closed World Assumption (CWA).

An inductive definition is a precise, well-understood informal language construct of mathematicians which makes this concept amenable for logical formalization. A natural, modular syntax for representing such inductive definitions is as a set of rules

$$
\forall \bar{x}(A \leftarrow \varphi)
$$

where $A$ is an atom of a defined predicate, $\leftarrow$ is called the definitional implication (to be distinguished from material implication) and $\varphi$ a FO formula which may contain (classical) negation. Such a rule set aims to define the defined predicates in the head in terms of the other, called open symbols. In $[8,9]$, the thesis was argued that the parameterized form of well-founded semantics (WFS) defined in [10] formalizes all common forms of inductive definitions in mathematics. This parameterized WFS constructs a unique, possibly three-valued interpretation for the defined predicates in terms of any given interpretation of the open symbols of the definition.

The above formal construct has historical roots in LP. The syntax and the parameterized WFS were presented first in [10], not as formal construct to represent definitions but as an extended datalog program. A logic program corresponds to a definition defining all its predicates. Given that inductive definitions include CWA, this view matches with the standard view of a logic program as a collection of clauses under CWA. An abductive logic program [1] corresponds to a definition whose open predicates are the abducible ones. A definition with open predicates might be viewed as a collection of clauses with a parameterized form of CWA, where the open symbols of the definition are unconstrained and the CWA of inductive definitions derives the defined predicates from the interpretation of the open symbols. 
Our KB language FO(ID) is obtained by integrating such definitions in FO. Our motivation for this is that, on the one hand, we believe that FO and its connectives and quantifiers $(\wedge, \neg, \forall, \ldots)$ are indispensable for KR. Extrapolating [11], we believe that any expressive KB language will have a sizable overlap with FO. (E.g., ASP constraints are a form of FO formulas in disguise.) On the other hand, expressing inductive definitions such as reachability and transitive closure is a well-known weakness of FO. Hence, it makes perfect sense to extend FO with inductive definitions. Formally, an FO(ID) theory is a set of FO axioms and definitions ${ }^{1}$. A model of such a theory is a (2-valued) structure satisfying all FO axioms and being a well-founded model of all definitions. Conceptually, FO(ID) can be seen as an expressive description logic using definitions for the TBox and FO for the ABox. It is also strongly related to fixpoint extensions of FO [12].

FO(ID) is a conceptually clean (read "non-hybrid") integration of FO and LP, and combines the strengths of both. In particular, inductive definitions are a precise natural non-monotonic construct from mathematics but they are also very useful for representing common sense knowledge. We already mentioned the relation to CWA. It follows that the methodologies for representing defaults and exceptions developed for LP under WFS, work also using FO(ID) definitions. As shown in [13], there is a straightforward modular mapping from logic programs under stable semantics into a sublogic of FO(ID). This means that methodologies for KR using this formalism can be emulated in FO(ID). Likewise for the KR methodologies of ALP: an abductive logic framework consisting of an abductive program and a set of FO constraints, can be viewed as an FO(ID) theory with one definition.

Inductive definitions include CWA but seem strongly related to another concept of common sense knowledge, namely causality. Recall that a definition defines a relation by describing how to construct it. Thus, such definitions implicitly describe a sort of mathematical construction processes which show strong similarity with causal ramification processes. In [14], we defined a logic for modeling non-deterministic probabilistic causality by extending definitions with causal rules with probabilities and disjunction in the head.

We thus argue that FO(ID)'s definition construct not only compensates for FO's weakness on expressing inductive definitions but also for FO's weakness on expressing common sense knowledge. Hence, FO(ID) might provide a solid theoretical underpinning for the recent attempts to integrate monotone and non-monotone logic, in particular the family of hybrid logics that combine logic programming with description logics [15].

\section{Reasoning in FO(ID)}

Several forms of inference for FO(ID) are under development. Most progress has been obtained for finite model expansion (MX). Model expansion is a sort of finite model generation in which the goal is to compute (finite) models $M$ of an input theory $T$ that expand a finite input structure $I$ interpreting an subvocabulary $\sigma$ (i.e., $M \models T$ and

\footnotetext{
${ }^{1}$ In [9], an FO(ID) theory is defined more generally, as a set of a boolean combinations of FO formulas and definitions.
} 
$\left.\left.M\right|_{\sigma}=I\right)$. Thus, the input structure $I$ fixes a finite domain and is useful to store data which are available in many MX-problems. In [16], MX was proposed as an alternative declarative programming paradigm that generalizes finite Herbrand model generation and offers some practical and theoretical advantages. Every MX problem in FO is an NP search problem; the same holds for every logic for which finite model checking is polynomial in the size of the domain. Inversely, MX in FO captures NP: for any NP class $\mathcal{C}$ of finite $\sigma$-structures, there exists an FO theory $T_{\mathcal{C}}$ such that $\sigma$-structure $I \in C$ iff $I$ can be expanded to a model of $T_{\mathcal{C}}$.

Several model generation or expansion systems are available for languages based on FO, such as aspps [13] and MXG [17]. Our group has developed the IDP system², an MX solver for a rich extension of full FO, including an order-sorted type system, inductive definitions, partial functions, arithmetic, existential quantifiers with numerical bounds and aggregates such as cardinality, minimum, maximum and sum. Though in principle none of these extensions increase the class of problems that can be solved using MX in FO [16], they do often considerably simplify the modeling task and increase the class of problems that can be solved in practice. For instance, reachability in the context of a finite domain can be expressed in FO, but not in a natural manner. On the other hand, it can easily be expressed by an inductive definition, and a solver able to natively handle such definitions is more efficient than a SAT solver on FO encodings of reachability.

To illustrate language and system, assume that company A wishes to take control over company B, by spending at most a fixed amount of say 100M buying shares in other companies. This is expressed in the following theory, together with the recursive definition of Controls $(a, b)$ and a definition for $\operatorname{Shares}(a, b, s)$ expressing that, after the purchases, $a$ has $s$ shares in $b$ :

$$
\begin{aligned}
& \text { Controls }(A, B) \\
& \operatorname{Sum}(\{c \mid \exists s(\operatorname{Buy}(A, b, s) \wedge c=s \times \operatorname{Cost}(b))\}) \leq 100 \\
& \left\{\begin{array}{l}
\forall a b\left(\text { Controls }(a, b) \leftarrow 50<\operatorname{Sum}\left(\left\{s \mid \begin{array}{l}
(z=a \vee \operatorname{Controls}(a, z)) \wedge \\
\operatorname{Shares}(z, b, s)
\end{array}\right\}\right)\right. \\
\forall a b s\left(\operatorname{Shares}(a, b, s) \leftarrow s=\operatorname{Sum}\left(\left\{s^{\prime} \mid \begin{array}{l}
\text { Buy }\left(a, b, s^{\prime}\right) \vee \\
\operatorname{IShares}\left(a, b, s^{\prime}\right)
\end{array}\right\}\right)\right.
\end{array}\right\}
\end{aligned}
$$

Note that it may be cheaper for A to buy shares in third companies than directly in B. IDP can solve this problem as an MX problem using an input structure specifying the initial shareholders in IShares and the cost of the shares in the function Cost.

The IDP system consists of a grounder that uses approximate reasoning to reduce grounding size and a propositional solver built on top of the minisat solver. In a series of experiments ${ }^{2}$, we compared IDP to a number of other MX and ASP systems over a range of different problems. More concretely, we considered (Lparse+)Clasp, DLV, MXG, aspps and IDP. The IDP system had the best performance on at least three aggregated measures: number of solved instances, number of instances solved in less than 10 seconds, and total time.

\footnotetext{
${ }^{2}$ A description of the IDP system and details of the experiments can be obtained via http://www.cs.kuleuven.be/ dtai/krr/LaSh.html.
} 


\section{Conclusion}

We believe that the best strategy to consolidate LP's contributions to KR and to certify LP's long term future as a KR language, is to show what it contributes to classical logic and to integrate it with the latter. This is what FO(ID) achieves. We stressed the strong relation between inductive definitions and concepts of common sense reasoning such as CWA and causality. Thus, the "logic programs", i.e., the definitions, in FO(ID) compensate for FO's weakness on representing inductive definitions and common sense knowledge. The feasibility of using this logic for problem solving is demonstrated by the model expansion system IDP. This system supports a rich extension of FO(ID) and, by integrating state-of-the-art technologies from SAT and ASP, it is competitive with the best ASP systems.

\section{References}

1. Kakas, A.C., Kowalski, R., Toni, F.: Abductive logic programming. Journal of Logic and Computation 2(6) (1992) 719-770

2. Marek, V.W., Truszczyński, M.: Stable models and an alternative logic programming paradigm. In Apt, K.R., Marek, V.W., Truszczyński, M., Warren, D.S., eds.: The Logic Programming Paradigm: a 25-Year Perspective. Springer-Verlag (1999) 375-398

3. Kautz, H., Selman, B.: Pushing the envelope: Planning, propositional logic, and stochastic search. In: AAAI'96. (1996)

4. Prasad, M., Biere, A., Gupta, A.: A survey of recent advances in sat-based formal verification. Intl. Journal on Software Tools for Technology Transfer (STTT) 7(2) (2005)

5. Stuckenschmidt, H.: Partial matching using approximate subsumption. In: AAAI'07. (2007)

6. Gelfond, M., Lifschitz, V.: The stable model semantics for logic programming. In: International Joint Conference and Symposium on Logic Programming (JICSLP'88), MIT Press (1988) 1070-1080

7. Clark, K.L.: Negation as failure. In Gallaire, H., Minker, J., eds.: Logic and Databases. Plenum Press (1978) 293-322

8. Denecker, M., Bruynooghe, M., Marek, V.: Logic programming revisited: Logic programs as inductive definitions. ACM Transactions on Computational Logic 2(4) (2001) 623-654

9. Denecker, M., Ternovska, E.: A logic of non-monotone inductive definitions. Transactions On Computational Logic (TOCL) 9(2) (2008)

10. Van Gelder, A.: The alternating fixpoint of logic programs with negation. Journal of Computer and System Sciences 47(1) (1993) 185-221

11. Moore, R.: The role of logic in knowledge representation and commonsense reasoning. In: AAAI' 82. (1982) 428-433

12. Libkin, L.: Elements of Finite Model Theory. Springer (2004)

13. East, D., Truszczyński, M.: Predicate-calculus-based logics for modeling and solving search problems. ACM Trans. Comput. Log. 7(1) (2006) 38-83

14. Vennekens, J., Denecker, M., Bruynooghe, M.: Representing causal information about a probabilistic process. In: JELIA’06. Volume 4160 of LNCS., Springer (2006) 452-464

15. Eiter, T., Lukasiewicz, T., Schindlauer, R., Tompits, H.: Combining answer set programming with description logics for the semantic web. In: KR'04. (2004)

16. Mitchell, D.G., Ternovska, E.: A framework for representing and solving NP search problems. In : AAAI05 (2005). 430-435

17. Mitchell, D., Ternovska, E., Hach, F., Mohebali, R.: Model expansion as a framework for modelling and solving search problems. Technical Report TR2006-24, Simon Fraser University (2006) 